\title{
A Protocol to Systematic Radiographic Assessment of Primary Total Knee Arthroplasty
}

\author{
Ahmed A Khalifa (D) \\ Arun B Mullaji $\mathbb{D}^{2}$ \\ Alaa M Mostafa' \\ Osama A Farouk iD ${ }^{3}$ \\ 'Orthopaedic Department, Qena Faculty \\ of Medicine and University Hospital, \\ South Valley University, Qena, Egypt; \\ ${ }^{2}$ Orthopaedic Department, Breach \\ Candy Hospital, Mumbai, India; \\ ${ }^{3}$ Orthopaedic and Traumatology \\ Department, Assiut University Hospital, \\ Assiut, Egypt
}

\begin{abstract}
Although total knee arthroplasty (TKA) is considered one of the most successful procedures, however, a subset of patients are unsatisfied with the results, even with the introduction of new technologies and implant designs. Radiological assessment of TKA is still considered the most prevalent imaging modality for evaluating the knee joint pre-and postoperatively. Assessment of various angles and indices which could be measured in different radiographic views of the knee provides valuable information about the alignment of the entire limb and the individual prosthetic components, more so in the light of recent nuanced concepts of technique, alignment, and balance. This review article aims to present a comprehensive yet systematic approach to the most useful radiographic parameters for assessing the knee preoperatively and post-TKA by explaining the tools and techniques used for measuring various angles, indices and ratios in the coronal, sagittal and axial planes for diagnosis, preoperative planning, postoperative assessment, and routine follow-up. The protocol we followed in this review entailed first reporting the possible applications and software which could help in measuring these variables, then we mentioned the required series of knee radiographs. For the desired variables, we divided the assessment according to each plane, and in each, we reported the optimum position of the desired radiographic view followed by determining the axis and lines which will later form the desired angles to be measured; finally, we collected all the measurements in a table with the native knee values and the most accepted values after TKA.
\end{abstract}

Keywords: assessment, knee radiograph, postoperative, total knee, replacement, arthroplasty

\section{Introduction}

The success of total knee arthroplasty (TKA) depends on many factors related to the patients, the surgeon, and the implant. ${ }^{1-3}$ The pivotal role of the surgeon, besides making the key decision for a knee replacement in conjunction with the patient, includes pre-operative planning (usually performed on plain knee radiographs either manually or using computerized software), executing this plan intraoperatively, assessing the postoperative radiological outcome and whether the objectives of limb and component alignment were achieved, and reviewing the patient and radiographs at follow-up visits to confirm that the improvements are maintained over time. ${ }^{1}$ Plain knee radiographs are still considered the primary imaging study for fulfilling these objectives. ${ }^{4}$ Other advanced imaging modalities such as computed tomography (CT) or magnetic resonance imaging (MRI) are typically used in specific situations such as complex deformities and when patient-specific instrumentation and/or custom implants are deployed. ${ }^{1}$ Scoring and evaluation systems have been developed for clinical and radiographic assessment after TKA to uniformly report results to facilitate comparisons of different techniques and implants; one commonly used is the scoring system 
developed by the Knee Society (KS), which incorporates data regarding alignment and component positions. ${ }^{2,5}$ However, these systems had been criticized for measuring a limited number of variables and do not take into consideration the more recent and nuanced concepts of surgical techniques, alignment and balancing, ${ }^{6}$ as well as recent studies describing variations in femoral and tibial phenotypes. ${ }^{7}$ A more detailed radiographic assessment may facilitate research into the variability of patient satisfaction after TKA.

The purpose of this review was to summarize the essential angles, distances, and ratios that should be radiographically assessed pre-, immediate post-TKA, and during follow-ups using a systematic approach. This review will not comment on the identification of implant type, quality of fixation, or how to detect complications. Evaluation will be described in the three standard planes: coronal, sagittal, and axial.

\section{Some Complementary Software and Applications Available}

1. IC Measure Digital software ${ }^{8,9}$ (The Imaging Source Europe $\mathrm{GmbH}$, Bremen, Germany) is a free downloadable program for PCs; it can measure angles and lengths.

2. Surgimap Spine software ${ }^{10}$ (Nemaris Inc, New York, NY) can be freely downloaded and is compatible with PC and Android or IOS platform smart devices and can also measure angles and lengths.

3. Protractor applications are free for Android and IOS smartphones for angle measurements. ${ }^{8}$

\section{Which Radiographic Views Should Be Obtained?}

Many protocols have been suggested for obtaining radiographic views for TKA evaluation; according to Meneghini et al. ${ }^{2}$ Based on a modification of the KS assessment protocol, three views are essential, first: a weight-bearing AP knee view, second: a lateral knee view, and lastly a patellofemoral assessment view. The protocol we recommend in this review is the same as that mentioned by Meneghini et al. ${ }^{2}$ However, for the AP view, a long film (when feasible) taken standing and including the hip to the ankle; Hip Knee Ankle film (HKA), is preferred especially in the assessment of complex cases. ${ }^{1}$ To these views, we have added the kneeling view to evaluate distal femoral rotation. ${ }^{11}$
Controversy still exists about whether HKA radiographs or short film AP knee radiographs should be used to assess implant position and coronal alignment after TKA; however, many studies have demonstrated that the short AP radiographs are insufficient for adequate assessment. ${ }^{12,13}$

\section{Essential Precautions While Evaluating the Radiographs}

1. Avoid malrotation of the limb: Radtke et al reported a significant impact of limb malrotation on the radiographic evaluation of the whole limb and individual component alignment after TKA, where internal rotation can lead to a false impression of valgus alignment of the limb in the coronal plane and increase in the individual component alignment angles and vice versa. ${ }^{14}$ Jiang and Insall showed that the lower limb's malrotation between $20^{\circ}$ of internal and external rotation would change the coronal alignment by about $2.5^{\circ} .{ }^{15}$

2. Ensure that the patient is weight-bearing and not supine: A weight-bearing film provides more information regarding the entire lower limb alignment and laxity of collateral ligaments, which may not be detected in non-weight-bearing radiographs. Deep et al found that the HKA angle changes significantly with posture changes as the knee becomes more varus by about $2.2^{\circ}$ on standing compared to the supine position. ${ }^{16}$

3. Magnification: For proper measurements and planning, the magnification of the radiographs should be known, or at least the radiographs should have a calibrated reference or a marker (like a coin) of known dimensions. This will increase the accuracy of preoperative templating, prediction of the implant sizes, and assessment of distances or ratios.

In the following, we will describe the detailed protocol of assessment divided according to the planes into coronal, sagittal, and axial; in each, we will mention the radiographic film criteria, required axes and lines to be defined and the possible angles to be measured.

\section{Coronal (c) Plane Evaluation (AP Radiographs) \\ Patient Positioning and Film Criteria}

For a proper short AP view, the knee must be fully extended (or as maximally as possible), with the patient standing, the 
patella facing forward, the x-ray beam centered over the knee, the distance between both feet should be at least $30 \mathrm{~cm},{ }^{17}$ and include the lower part of the distal femur, knee joint, and proximal part of the tibia and fibula. ${ }^{5}$ To get an appropriate HKA film, both limbs should be in a neutral rotation; usually, half of the lesser trochanter should be visible in profile (the whole of the lesser trochanter can be visualized in a radiograph of an externally rotated lower limb and vice versa), and the medial half of the fibular head should overlap with the tibial metaphysis (an internally rotated leg will show less overlapping). ${ }^{15,18}$

\section{Coronal (c) Plane Measurements 19,20}

Axis and lines determination (Figure 1A): for any long bone, its mechanical axis is the line between the center of proximal and distal articulations; its anatomical axis is the line that passes through the middle of the shaft. ${ }^{19,21,22}$

1. Femoral anatomical axis (cFAA): In a short AP film, it is a line connecting a point midway between the medial and lateral cortices as far proximally as the film allows to a similar point $10 \mathrm{~cm}$ proximal to the joint line. ${ }^{5,19}$ In a long HKA film, it is a line connecting the midpoint of the isthmus to a similar midpoint $10 \mathrm{~cm}$ proximal to the joint line. $^{19}$

2. Femoral mechanical axis (cFMA): is the line between the femoral head center and the center of the trochlea of a native knee or trochlear notch of the femoral component after TKA.

3. Tibial anatomical axis (cTAA): In a short AP film, it is a line connecting a point midway between the medial and lateral cortices $10 \mathrm{~cm}$ distal to the joint line to a similar point distally as far as the film allows. ${ }^{5,19}$ In a long HKA film, a line is formed by connecting the midpoint of the distal third of the tibia to the center of the tibia $10 \mathrm{~cm}$ distal to the joint line. ${ }^{16}$ Some authors support the idea that the TAA coincides with the mechanical one, ${ }^{22}$ according to Paley, the mechanical and anatomical axes are parallel but not the same. ${ }^{19}$

4. Tibial mechanical axis (cTMA): is the line between the talus center and the center of the tibial plateau of a native knee or the tibial component of the TKA.
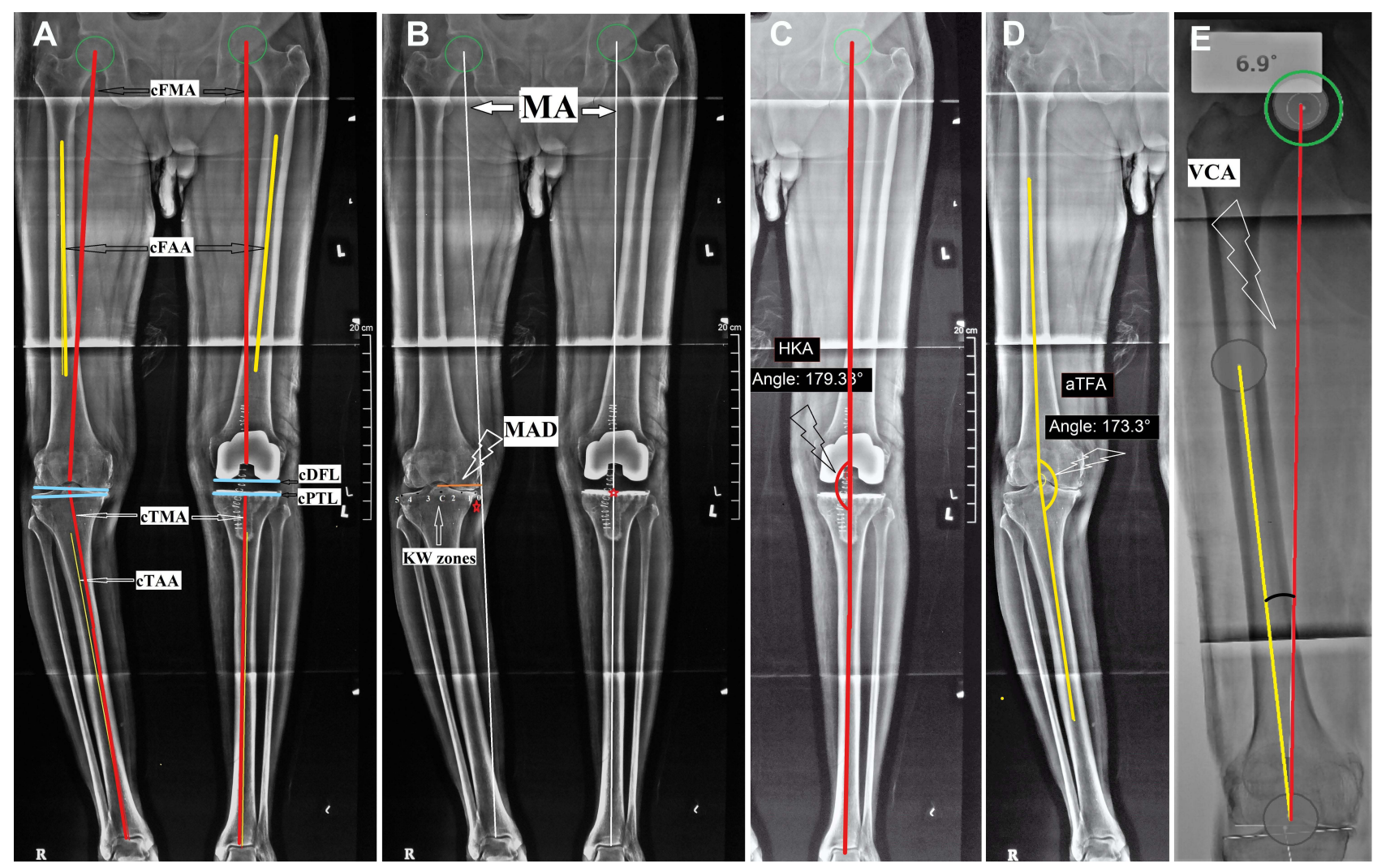

Figure I Assessment in the coronal plane (AP radiograph).

Notes: (A) defining the axes; c, coronal; FMA, femoral mechanical axis; FAA, femoral anatomical axis; DFL, distal femoral line; PTL, proximal tibial line; TMA, tibial mechanical axis; TAA, tibial anatomical axis; (B) lower limb mechanical axis (MA); MAD, mechanical axis deviation; KY, Kennedy and White; (C) HKA, hip, knee to ankle angle; (D) aTFA, anatomical tibiofemoral angle; (E) VCA, valgus correction angle. 
5. Distal femoral line (cDFL): is a line tangential to the most distal points on the convexity of the two femoral condyles of the native knee or the femoral component of the TKA. ${ }^{19}$

6. Proximal tibial line (cPTL) is drawn across the flat or concave aspect of the two tibial plateaus' subchondral line in a native knee or the line at the base of the tibial component in TKA. ${ }^{18}$

Using the above axes and lines, the following variables are measured: $:^{2,5,19,20}$

(a) Mechanical axis deviation (MAD) (Figure 1B): is measured as a perpendicular line drawn from Maquet's line (which is the mechanical axis (MA) of the lower limb extending from femoral head center to center of talar dome $)^{23,24}$ to the center of the knee; it normally measures $9.7 \pm 6.8 \mathrm{~mm} .{ }^{19,20}$ The relationship between MA and the center of the knee, identified as MAD, is linear; for every $1^{\circ}$ of valgus or varus, the MA moves for about $5 \mathrm{~mm}$ away from the knee's center. The tibial plateau has been divided into seven zones (from medial to lateral: zones $0,1,2, \mathrm{C}, 3,4,5)$ and the passage of the MA through the knee can be described according to which of these Kennedy and White zones (KW zones) the MA lies within. ${ }^{25-27}$

(b) The hip-knee-ankle angle (HKA) (Figure 1C): is the medial angle between the cFMA and the cTMA, indicating the limb's mechanical alignment. A neutral mechanical alignment is varus $1.3 \pm$ $1.3^{\circ},{ }^{19}$ and an angle of $0 \pm 3^{\circ}\left(180 \pm 3^{\circ}\right)$ is the classic safe target for alignment after TKA. ${ }^{28}$

(c) Anatomical tibiofemoral angle (aTFA) (Figure 1D): the medial angle between the cFAA and the cTAA, indicating the limb's anatomical alignment. Normally it measures valgus $7^{\circ} \pm 1.4 .^{19}$ A valgus angle between $2.4^{\circ}$ and $7.2^{\circ}$ after TKA is considered neutral anatomic alignment. ${ }^{1}$

(d) Valgus correction angle (VCA) ${ }^{29}$ (Figure 1E): the angle between the cFMA and cFAA determines the distal femoral cut angle in TKA. It had been reported to range between 3 and 11 degrees. ${ }^{29}$

(e) Mechanical lateral distal femoral angle (mLDFA) (Figure 2A): is the lateral angle formed between the cFMA and cDFL; normally, it measures $87.8^{\circ} \pm$ 1.6. ${ }^{19,20}$ The femoral component position in the coronal plane should be placed at $90^{\circ} \pm 3$ to the
cFMA or according to the preoperatively measured VCA. ${ }^{29,30}$

(f) Anatomical lateral distal femoral angle (aLDFA) (Figure 2B): is the lateral angle between the FAA and cDFL. Normally it ranges from $79^{\circ}$ to $83^{\circ} .^{19} \mathrm{An}$ angle of $85^{\circ} \pm 2$ is considered acceptable after TKA. ${ }^{30}$

(g) Medial proximal tibial angle (MPTA) (Figure 2C): (The $\beta$ tibial angle in the KS scoring system) is the medial angle formed between the TMA and cPTL; normally, it measures $87.2^{\circ} \pm 1.5 .^{19,20}$ It reflects the tibial component position in the coronal plane, an angle between 0 to varus 3 degrees $\left(90^{\circ}\right.$ to $\left.87^{\circ}\right)$ being considered safe. ${ }^{31}$

(h) Joint line height $(\mathrm{cJLH})^{32,33}$ (Figure 2D): The distance from the joint line (the line connecting the medial and lateral joint space or the line connecting the most distal points of the medial and lateral femoral condyles) to a fixed bony landmark, proximally as the medial femoral epicondyle or distally as the fibular head. It is usually 25 to $28 \mathrm{~mm}$ from the medial femoral epicondyle and 10 to $14 \mathrm{~mm}$ from the fibular head. ${ }^{32,33}$

(i) Leg length difference (LLD) ${ }^{34}$ (Figure 2E): is measured as the difference in the length of a line connecting the femoral head center to the center of the talus in the two limbs.

(j) Component size: Ideally, the components should replicate the patient's anatomy if possible. The femoral component should lie flush with the margins of the femoral condyles medially and laterally in the AP radiograph. The margins of correctly sized tibial components should likewise be flush with the medial and lateral cortices in AP views. $^{35,36}$

\section{Sagittal (s) Plane Evaluation (Lateral Radiographs)}

\section{Patient Positioning and Film Criteria}

For a standard short film lateral knee view, the knee is flexed $30^{\circ}$; the patella should be perpendicular to the cassette, with the lower leg being parallel to the radiological table Obtaining as much as possible of the tibial and femoral shafts within the film is essential to detect any excessive bowing or extra-articular deformity (EAD). ${ }^{19}$ An accurate lateral view of the knee should have no overlap between the medial and lateral femoral condyles. To 


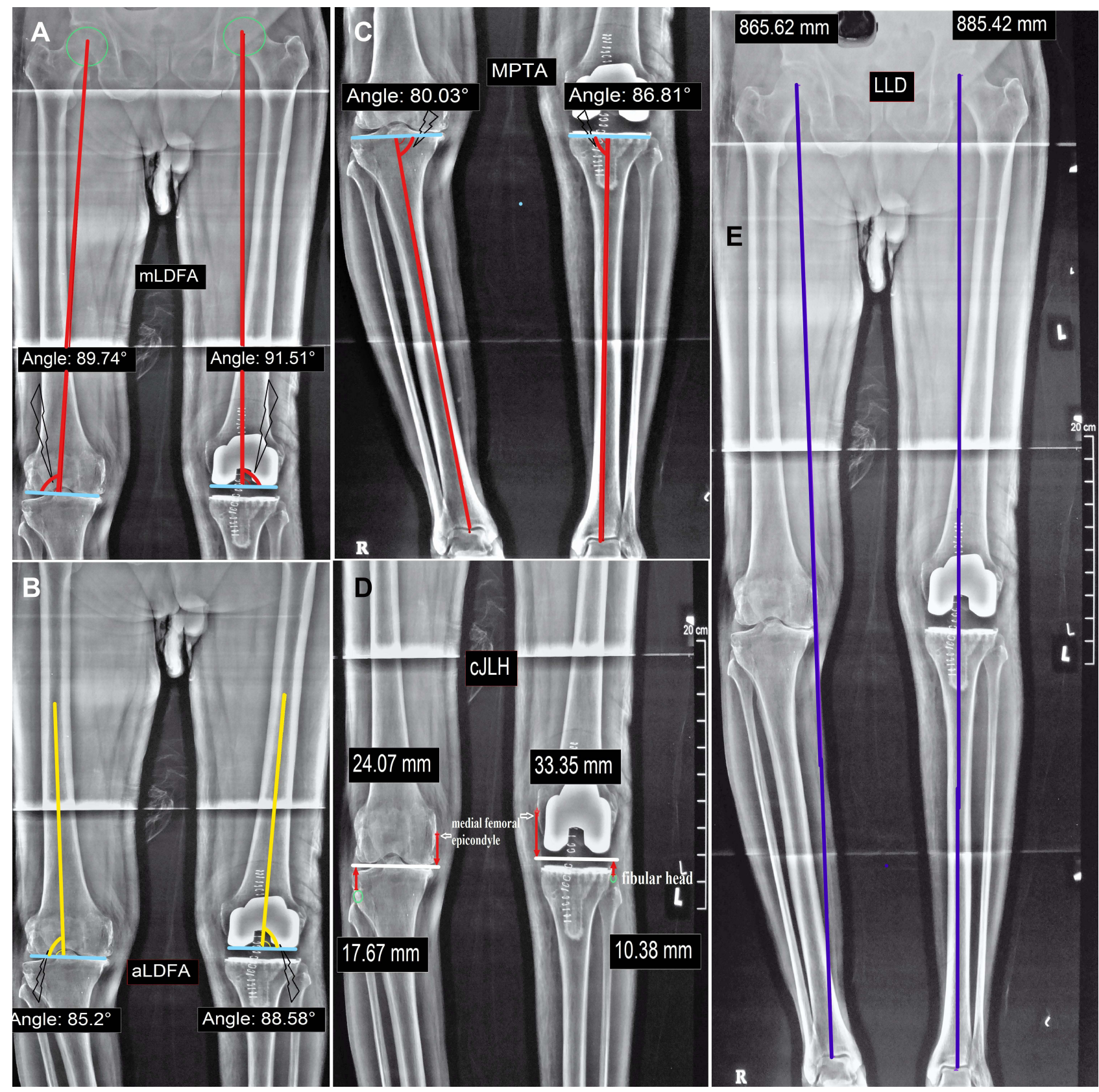

Figure 2 Assessment in coronal plane continued.

Notes: (A), mLDFA, mechanical lateral distal femoral angle; (B) aLDFA, anatomical lateral distal femoral angle; (C) MPTA, medial proximal tibial angle; (D) cJLH, coronal joint line height; (E) LLD, leg length discrepancy.

obtain a lateral view of the whole femur (including the femoral heads), Chung et al positioned the patient's thigh on a 17 x 17-inch digital detector with the x-ray beam angled at $15^{\circ}$ cranially. ${ }^{37}$

\section{Sagittal (s) Plane Measurements ${ }^{19,20}$}

Axis and lines determination (Figure 3A)
1. Femoral anatomical axis (sFAA): on the short film, it is a line obtained by joining a point at the middle of the femoral shaft (as proximal as possible in the film) with a second point placed at $10 \mathrm{~cm}$ proximal to the joint line in the middle of the femoral shaft. On a long film (showing the whole femur), the sFAA is obtained by drawing a line through the femoral shaft's proximal, middle, and distal centers; $;{ }^{19}$ This produces a segmented 


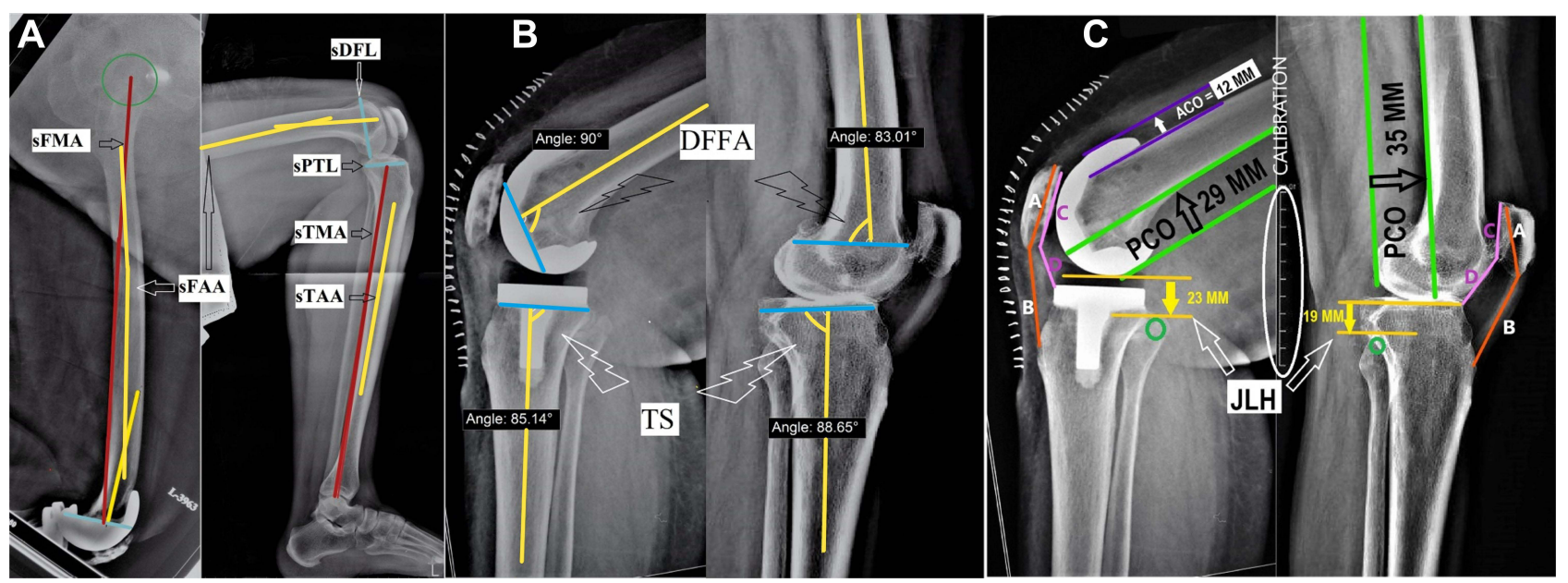

Figure 3 Assessment in the sagittal plane (Lateral radiograph).

Notes: (A) defining the axes; s, sagittal; FMA, femoral mechanical axis; FAA, femoral anatomical axis; DFL, distal femoral line; PTL, proximal tibial line; TMA, tibial mechanical axis; TAA, tibial anatomical axis; (B) DFFA, distal femoral flexion angle; TS, tibial slope; (C) ACO, anterior condylar offset; PCO, posterior condylar offset; JLH, joint line height; Insall Salvati index = length of line B/ length of line A, Caton-Deschamps Index = length of line C/length of line D.

line representing the sagittal femoral curve (normally between $4^{\circ}$ and $\left.9^{\circ}\right) .^{37}$

2. Femoral mechanical axis (sFMA) (in a long film): it is the line drawn from the femoral head center to the center of the distal femur or the center of the femoral component. ${ }^{19}$

3. Tibial anatomical axis (sTAA): is formed by connecting the most distal mid-point of the tibial shaft with a point located $10 \mathrm{~cm}$ distal to the knee joint line. ${ }^{20}$

4. Tibial mechanical axis (sTMA): is the line between the center of the talus and the center of the tibial plateau or the tibial component (if the film shows the whole tibia). ${ }^{19}$

5. Distal femoral line (sDFL): the distal femoral condyles are circular, so the DFL in the sagittal plane can be drawn as a straight line connecting two points where the condyles merge with the distal femoral metaphysis (for a native knee). For a femoral component after TKA, this line can be drawn either at the distal femur's resection line or the line tangent to the intercondylar box (if a posterior stabilized implant was used).

6. Proximal tibial line (sPTL): is a line along the flat subchondral area of the tibial plateau for the native knee or a line tangent to the undersurface of the tibial component after TKA.

Using the above axes and lines, the following variables are measured: $:^{2,5,19,20}$ (a) Distal Femoral flexion angle (DFFA) (Figure 3B): is the posterior angle between the sFAA and the sDFL; normally, it is $83^{\circ} \pm 4 .{ }^{20}$ It reflects the flexion or extension position of the femoral component (angle of 90 degrees is considered neutral, $>90$ degrees considered an extension, and $<90$ considered flexion. An angle from $90^{\circ}$ to $87^{\circ}$ is considered as accepted after TKA). ${ }^{24}$

(b) Tibial slope (TS) (Figure 3B): is the posterior angle between the sTAA and the SPTL, represented as the $\sigma$ angle in the KS scoring system. The normal tibial slope is $81^{\circ} \pm 3 .{ }^{20}$ Some authors advocate keeping the TS between $0^{\circ}$ and $7^{\circ}$ during TKA; however, this value may differ according to various implant designs. $^{38,39}$

(c) The posterior condylar offset (PCO) (Figure 3C): measured as the thickness of the posterior femoral condyles (either of the native femur or the femoral component after TKA) projected posteriorly from a tangent line over the posterior femoral cortex. It should be restored during TKA as a PCO decreases after surgery will affect knee flexion; for every $2 \mathrm{~mm}$ decrease in PCO, flexion is reduced by about $12.2^{\circ} .{ }^{40}$

(d) The anterior condylar offset (ACO) (Figure 3C): measured as the maximum thickness of the anterior condyles of the native knee or the anterior flange of the femoral component after TKA projected 
anteriorly from a tangent line to the anterior femoral cortex (serves as an indication of patellofemoral overstuffing). ${ }^{41}$ Miller et al reported that following an increase of the ACO between 2 and $4 \mathrm{~mm}$, the flexion decreases by $1.8^{\circ}$ and $4.4^{\circ}$, respectively. ${ }^{42}$

(e) Patellar height (can be measured using different techniques): ${ }^{43}$

i. Insall-Salvati Index (ISI) (Figure 3C): it is the ratio between the patellar tendon length (measured from its origin at the patellar lower pole to its insertion in the tibial tuberosity, line B) and the length of the patella (measured as the longest diagonal line across the patella, line A). Normally, the ratio is about 1.02 , with a variation of less than $20 \%$. A ratio greater than 1.2 indicates a patella Alta, and if lower than 0.8 indicates a patella Baja. ${ }^{44}$

ii. Caton-Deschamps Index (CDI) (Figure 3C): it is the ratio between the length of the patellar articular surface (line C) and the length of the distance from the inferior pole of the patella to the superior margin of the tibial plateau (line D). The normal value is $1.0(0.8-1.2)$, a ratio higher than 1.3 indicates a patella Alta, and lower than 0.7 suggests a patella Baja. ${ }^{45}$ The use of the ISI is theoretically favored over the CDI because it is not adversely affected by the position of the joint line, which can be altered by surgery. ${ }^{46}$

iii. The joint line height (sJLH) (Figure 3C): is measured as the perpendicular distance from the weight-bearing parallel surface of the tibial plateau or the most distal points of the femoral component to the tip of the fibular head. ${ }^{47}$

(f) Femoral notching: Anterior femoral cortical notching can be visualized in a lateral view. It acts as a stress riser at the anterior femoral cortex and may increase supracondylar fracture risk. ${ }^{48}$

(g) Component size: An oversized femoral component can overstuff the patellofemoral joint or create a tight flexion gap to reduce the range of flexion. An undersized femoral component can cause instability in flexion due to a large flexion gap. ${ }^{49}$ The tibial component should not overhang posteriorly to avoid post TKA flexion deformity or any impingement. ${ }^{50}$

\section{Axial (a) Plane Evaluation (Axial and Special View Radiographs) Patient Positioning and Film Criteria}

There are variations in positions described for axial plane assessment of the patellofemoral joint, ${ }^{51}$ the most commonly used one being Merchant's view where the patient is supine on the radiological table with the knees flexed at $90^{\circ}$, and the cassette is placed proximally at the tibial shins. Both knees are exposed simultaneously with the beam directed from proximal to distal at $30^{\circ}$ from the horizontal. ${ }^{52}$

The kneeling view for distal femoral (and femoral component) rotational assessment and the technique for obtaining this view was described by Takai et al. ${ }^{11}$ The patient is seated on the edge of a radio-transparent table with the knee flexed to $90^{\circ}$, and the beam is passed from posterior either perpendicular to the tibial shaft or inclined $15^{\circ}$ to the horizontal plane from inferiorly. The resultant radiograph is a posterior-anterior view of a flexed knee showing the detailed anatomy of the distal femur.

\section{Axial Plane Measurements ${ }^{1,53}$}

Axis and lines determination (Figure 4)

1. Anterior femoral condylar line (AFCL): line passing tangent to the anterior femoral condyles of a native distal femur or the femoral component after TKA.

2. Patellar axis (PA): line passing from the medial to the lateral poles of the patella (equator).

3. Posterior condylar line (PCL) (in kneeling view): line passing tangentially to the posterior femoral condyles of a native femur or of the femoral component after TKA.

4. Transepicondylar axis (TEA) (in kneeling view): it could be either the anatomical axis (aTEA) represented by a line connecting the medial and lateral femoral epicondyles, or the surgical axis (sTEA) represented by a line connecting the medial sulcus with the lateral epicondyle.

\section{Patellofemoral Joint Measurements $2,5,53,54$}

1. Sulcus angle (measured in the native knee) (Figure 4A): is the angle formed between the lateral and medial anterior femoral condyles at the femoral sulcus trough; its normal value is $135^{\circ} \pm 10$, with an 


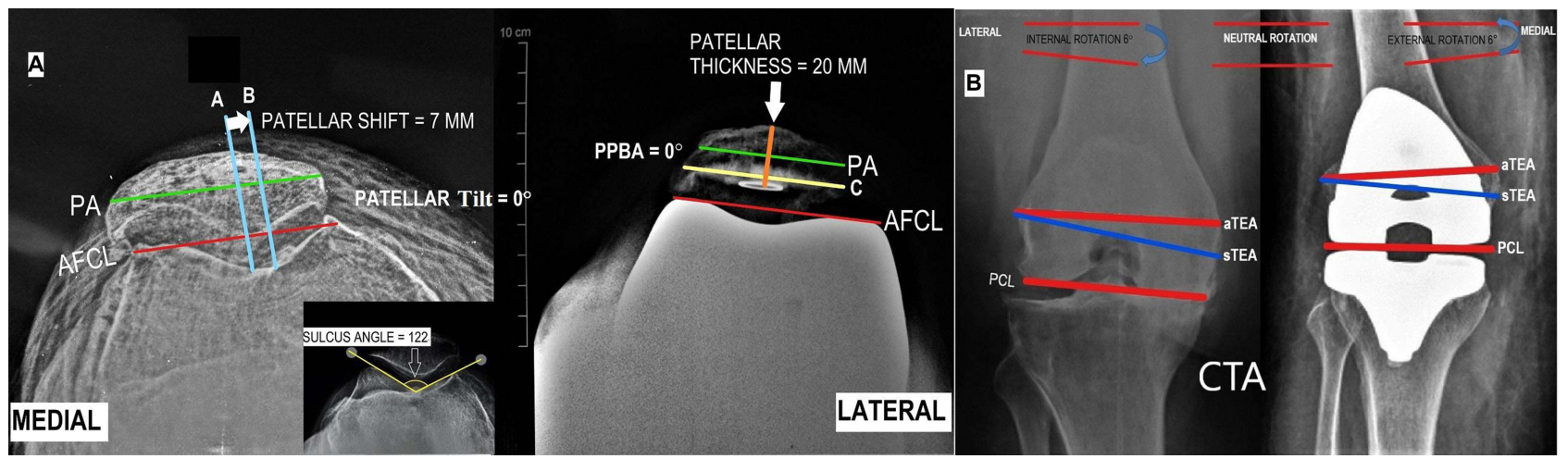

Figure 4 Assessment in the axial plane (special views).

Notes: (A) Merchant view; PA, patellar axis; AFCL, anterior femoral condylar line; PPBA, patellar prosthesis-bone angle; (B) kneeling view; CTA, condylar twist angle; aTEA, anatomical transepicondylar axis; sTEA, surgical transepicondylar axis; PCL, posterior condylar line.

increased angle indicating trochlear dysplasia and an increased incidence of patellar instability. ${ }^{55}$

2. Patellar tilt: is the angle between the AFCL and the PA line for a native or unresurfaced patella (represented by the bone prosthesis interface line in a resurfaced patella after TKA); the patella should lie at the center of the trochlear groove in Merchant's view; if the patella is tilted laterally, the angle is positive (this is considered normal up to $5^{\circ}$ ) if the angle is $>5^{\circ}$ it indicates patellar instability, ${ }^{4}$ while if it is $0^{\circ}$ or negative the patellar tilt is abnormal. A small or negative angle indicates a subluxation or a dislocation. It corresponds to $(\gamma)$ angle in the KS scoring system. ${ }^{17}$

3. The patellar thickness: is the vertical distance from the patella's anterior cortex to the deepest point of the patellar articular surface. Increased patellar thickness can alter the tracking and reduce the range of flexion; ${ }^{56}$ Benges and Scott showed in a study on cadavers that the flexion decreased by $3^{\circ}$ for every $2 \mathrm{~mm}$ incrementation of the patellar thickness. ${ }^{57}$

4. Patellar shift: is the mediolateral position of the patella measured as the horizontal distance between two vertical lines; the first line is passing through the medial ridge of the patella (line A) and another line passing through the deepest part of the trochlear groove (line B). Patella should be positioned on the medial two-thirds of the trochlear groove. ${ }^{58}$ It corresponds to $(\delta)$ in the KS scoring system. ${ }^{2}$ Chia et al reported that a preoperative lateral patellar shift of more than $3 \mathrm{~mm}$ was an independent risk factor for patellar maltracking after TKA. ${ }^{59}$
5. Patellar prosthesis-bone angle (PPBA): is the angle formed between the PA of the patella's remnant and the tangent to the bone-implant interface (line $\mathrm{C}$ ). The patellar prosthesis-bone angle can determine an asymmetrical bone cut of the patella during resurfacing.

\section{Condylar Twist Angle (CTA) ${ }^{11,60}$}

In the kneeling view, it is measured as the angle between the PCL and the aTEA (if aTEA cannot be identified, the sTEA can be used instead) (Figure 4B). It is used to determine the amount of preoperative distal femoral torsion of a native femur or the femoral component rotation after TKA. The rotational direction will be determined by the direction of rotation of the PCL in relation to the TEA. The preoperative CTA can differ greatly according to the arthritic process or ethnic differences; however, the postoperative CTA should be neutral (PCL parallel to aTEA) or between $3^{\circ}$ and $4^{\circ}$ of external rotation if measured in reference to the sTEA. ${ }^{11,61}$

All the previously mentioned parameters are listed in (Table 1) (the measurements which could not be performed either pre- or post-TKA, their spaces are blocked) which may serve as a guide for the surgeon for preoperative as well as post-TKA radiographic assessment. Although plain radiographs play the main role in the diagnosis and assessment of patients undergoing TKA, however, it is subject to human errors. Advanced imaging studies like CT and MRI may be needed in specific situations.

\section{Conclusion}

In this review, we followed a systematic protocol for knee radiographic assessment pre- and post-TKA; we tried to collect all the possible commonly reported variables (angles, ratios, and indices) needed for preoperative planning and 
Table I Radiographic Parameters for Knee Assessment Pre, Post, and for Follow-Up of TKA

\begin{tabular}{|c|c|c|c|c|c|}
\hline \multirow[t]{2}{*}{ Parameters } & \multirow{2}{*}{$\begin{array}{l}\text { Pre- } \\
\text { OP }\end{array}$} & \multirow{2}{*}{$\begin{array}{l}\text { Post- } \\
\text { OP }\end{array}$} & \multirow{2}{*}{$\begin{array}{l}\text { Follow } \\
\text { Up }\end{array}$} & \multicolumn{2}{|c|}{ Normal Values } \\
\hline & & & & Native Knee & TKA \\
\hline \multicolumn{6}{|c|}{ A-CORONAL PLANE (AP radiograph) } \\
\hline HKA & & & & $1.3 \pm 1.3^{\circ 19}$ & $0 \pm 3^{\circ}\left(180 \pm 3^{\circ}\right)^{28}$ \\
\hline VCA & & & & 3: $11^{29}$ & \\
\hline aTFA & & & & Valgus $7^{\circ} \pm 1.4^{19}$ & Valgus $2.4^{\circ}: 7.2^{\circ 1}$ \\
\hline mLDFA & & & & $87.8^{\circ} \pm 1.6^{19,20}$ & $90^{\circ} \pm 3^{29,30}$ \\
\hline aLDFA & & & & $79^{\circ}$ to $83^{\circ 19}$ & $85^{\circ} \pm 2^{30}$ \\
\hline MPTA & & & & $87.2^{\circ} \pm 1.5^{20}$ & $90^{\circ}: 87^{\circ 31}$ \\
\hline JLH & & & & $\begin{array}{l}\text { 25: } 28 \mathrm{~mm} \text { from the medial femoral } \\
\text { epicondyle-10: } 14 \mathrm{~mm} \text { from the fibular } \\
\text { head }^{32,33}\end{array}$ & Should reproduce the preoperative normal values \\
\hline LLD & & & & & \\
\hline \multicolumn{6}{|c|}{ B-SAGITTAL PLANE (Lateral radiograph) } \\
\hline TS & & & & $81^{\circ} \pm 3^{20}$ & $\begin{array}{l}0^{\circ}: 7^{\circ} \text { (may differ according to various implants } \\
\text { designs) }\end{array}$ \\
\hline DFFA & & & & $83^{\circ} \pm 4^{20}$ & $90^{\circ}: 87^{\circ 24}$ \\
\hline \multicolumn{6}{|l|}{ PCO } \\
\hline \multicolumn{6}{|l|}{ ACO } \\
\hline$|S|$ & & & & $1.2^{44}$ & $>1.2$ patella Alta, $<0.8$ patella Baja ${ }^{44}$ \\
\hline \multicolumn{6}{|l|}{ JLH } \\
\hline \multicolumn{6}{|c|}{ C-AXIAL PLANE (merchant, kneeling radiographs) } \\
\hline Sulcus angle & & & & $135 \pm 10^{\circ 55}$ & \\
\hline \multicolumn{6}{|l|}{ Patellar tilt } \\
\hline \multicolumn{6}{|l|}{$\begin{array}{l}\text { Patellar } \\
\text { thickness }\end{array}$} \\
\hline \multicolumn{6}{|l|}{ Patellar shift } \\
\hline \multicolumn{6}{|l|}{ PPBA } \\
\hline CTA & & & & $\begin{array}{l}\text { Varies according to deformity, arthritis, } \\
\text { ethnicity. }\end{array}$ & $\begin{array}{l}0^{\circ} \text { if measured in reference to the aTEA }-3^{\circ} \text { to } 4^{\circ} \text { of } \\
\text { external rotation if measured in reference to the } \\
\text { STEA. }{ }^{11,61}\end{array}$ \\
\hline
\end{tabular}

Abbreviations: AP, anteroposterior; HKA, hip, knee to ankle angle; VCA, valgus correction angle; aTFA, anatomical tibiofemoral angle; mLDFA, mechanical lateral distal femoral angle; aLDFA, anatomical lateral distal femoral angle; MPTA, medial proximal tibial angle; JLH, joint line height; LLD, leg length difference; TS, tibial slope; DFFA, distal femoral flexion angle; PCO, posterior condylar offset; ACO, anterior condylar offset; ISI, Insall Salvati index; PPBA, patellar prosthesis-bone angle; CTA, condylar twist angle.

postoperative evaluation. We believe that this review could guide surgeons for the proper technique of radiographic evaluation; however, each surgeon could define the useful variables for his daily practice. Furthermore, these angles and measurements could differ according to the design of the prosthesis used and the manufacturer's recommendations for implant positioning, as well as the alignment and balancing philosophy upon which the TKA is performed. All the 
reported measurements should be evaluated in conjunction with functional outcomes as well as patients satisfaction.

\section{Abbreviations}

TKA, total knee arthroplasty; CT, computed tomography; MRI, magnetic resonance imaging; HKA, Hip Knee Ankle; c, coronal; FAA, femoral anatomical axis; FMA, femoral mechanical axis; TAA, tibial anatomical axis; TMA, tibial mechanical axis; DFL, distal femoral line; PTL, proximal tibial line; MAD, mechanical axis deviation; MA, mechanical axis; aTFA, anatomical tibiofemoral angle; VCA, valgus correction angle; mLDFA, mechanical lateral distal femoral angle; aLDFA, anatomical lateral distal femoral angle; MPTA, medial proximal tibial angle; JLH, joint line height; LLD, leg length difference; s, Sagittal; DFFA, distal femoral flexion angle; TS, tibial slope; PCO, posterior condylar offset; ACO, anterior condylar offset; ISI, Insall-Salvati Index; CDI, Caton-Deschamps Index; a, axial; AFCL, anterior femoral condylar line; PA, patellar axis; PCL, posterior condylar line; TEA, transepicondylar axis; PPBA, patellar prosthesis-bone angle; CTA, condylar twist angle.

\section{Consent for Publication}

Authors declare that images are entirely unidentifiable, and there are no details on individuals reported in the published version.

\section{Author Contributions}

All authors made substantial contributions to conception and design, acquisition of data, or analysis and interpretation of data; took part in drafting the article or revising it critically for important intellectual content; agreed to submit to the current journal; gave final approval of the version to be published; and agreed to be accountable for all aspects of the work.

\section{Funding}

No funding was received for this manuscript.

\section{Disclosure}

The authors reported no conflicts of interest for this work.

\section{References}

1. Tanzer M, Makhdom AM. Preoperative planning in primary total knee arthroplasty. $J$ Am Acad Orthop Surg. 2016;24(4):220-230. doi:10.5435/JAAOS-D-14-00332

2. Meneghini RM, Mont MA, Backstein DB, Bourne RB, Dennis DA, Scuderi GR. Development of a modern knee society radiographic evaluation system and methodology for total knee arthroplasty. J Arthroplasty. 2015;30(12):2311-2314. doi:10.1016/j.arth.2015.05.049
3. Sardana V, Burzynski JM, Khan M, Stone N, Weening BS, Zalzal PK. Long-term functional outcomes and knee alignment of computer-assisted navigated total knee arthroplasty. Musculoskelet Surg. 2017;101(1):37-43. doi:10.1007/s12306-016-0442-z

4. Kumar N, Yadav C, Raj R, Anand S. How to interpret postoperative X-rays after total knee arthroplasty. Orthop Surg. 2014;6(3):179-186. doi:10.1111/os. 12123

5. Ewald FC. The knee society total knee arthroplasty roentgenographic evaluation and scoring system. Clin Orthop Relat Res. 1989;(248):9-12.

6. Riviere C, Iranpour F, Auvinet E, et al. Alignment options for total knee arthroplasty: a systematic review. OTSR. 2017;103 (7):1047-1056. doi:10.1016/j.otsr.2017.07.010

7. Hirschmann MT, Moser LB, Amsler F, Behrend H, Leclercq V, Hess S. Phenotyping the knee in young non-osteoarthritic knees shows a wide distribution of femoral and tibial coronal alignment. Knee Surg Sports Traumatol Arthrosc. 2019;27(5):1385-1393. doi:10.1007/s00167-019-05508-0

8. Khalifa A, Bakr H, Said E, Mahran M. Technical note on using intraoperative smartphone applications to adjust cup inclination angle during Total Hip Arthroplasty (THA). Archiv Bone Joint Surg. 2020;8(6):734-738. doi:10.22038/abjs.2020.44466.2217

9. Khalifa AA, Fayez M, Elkady H, Abdelaal AM, Elassal MA. The outcome of posterior-stabilized, rotating platform total knee arthroplasty at a minimum ten-year follow-up, a middle east institution experience. J Knee Surg. 2020. doi:10.1055/s-0040-1716850

10. Langella F, Villafane JH, Damilano M, et al. Predictive accuracy of surgimap surgical planning for sagittal imbalance: a cohort study. Spine. 2017;42(22):E1297-E1304. doi:10.1097/BRS.0000000000002230

11. Takai S, Yoshino N, Isshiki T, Hirasawa Y. Kneeling view: a new roentgenographic technique to assess rotational deformity and alignment of the distal femur. $J$ Arthroplasty. 2003;18(4):478-483. doi:10.1016/S0883-5403(03)00065-2

12. Stickley CD, Wages JJ, Hetzler RK, Andrews SN, Nakasone CK. Standard radiographs are not sufficient for assessing knee mechanical axis in patients with advanced osteoarthritis. $J$ Arthroplasty. 2017;32 (3):1013-1017. doi:10.1016/j.arth.2016.09.024

13. Dargel J, Pennig L, Schnurr C, Boese CK, Eysel P, Oppermann J. Ist die postoperative Ganzbeinaufnahme nach Knie-TEP-Implantation notwendig? [Should we use hip-ankle radiographs to assess the coronal alignment after total knee arthroplasty?]. Der Orthopade. 2016;45(7):591-596. German. doi:10.1007/s00132-016-3264-7

14. Radtke K, Becher C, Noll Y, Ostermeier S. Effect of limb rotation on radiographic alignment in total knee arthroplasties. Arch Orthop Trauma Surg. 2010;130(4):451-457. doi:10.1007/s00402-009-0999-1

15. Jiang CC, Insall JN. Effect of rotation on the axial alignment of the femur. Pitfalls in the use of femoral intramedullary guides in total knee arthroplasty. Clin Orthop Relat Res. 1989;(248):50-56.

16. Deep K, Eachempati KK, Apsingi S. The dynamic nature of alignment and variations in normal knees. Bone Joint J. 2015;97-B (4):498-502. doi:10.1302/0301-620X.97B4.33740

17. Hsu RW, Himeno S, Coventry MB, Chao EY. Normal axial alignment of the lower extremity and load-bearing distribution at the knee. Clin Orthop Relat Res. 1990;(255):215-227.

18. Tang WM, Zhu YH, Chiu KY. Axial alignment of the lower extremity in Chinese adults. $J$ Bone Joint Surg Am. 2000;82 (11):1603-1608. doi:10.2106/00004623-200011000-00014

19. Paley D. Radiographic assessment of lower limb deformities. In: Principles of Deformity Correction. Berlin, Heidelberg: Springer Berlin Heidelberg; 2002:31-60.

20. Paley D, Herzenberg JE, Tetsworth K, McKie J, Bhave A. Deformity planning for frontal and sagittal plane corrective osteotomies. Orthop Clin North Am. 1994;25(3):425-465. doi:10.1016/S0030-5898(20)31927-1

21. Paley D, Tetsworth K. Mechanical axis deviation of the lower limbs. Preoperative planning of uniapical angular deformities of the tibia or femur. Clin Orthop Relat Res. 1992;(280):48-64. 
22. Luo CF. Reference axes for reconstruction of the knee. Knee. 2004;11 (4):251-257. doi:10.1016/j.knee.2004.03.003

23. Maquet P. Biomécanique de la gonarthrose [Biomechanics of gonarthrosis]. Acta Orthop Belg. 1972;38(Suppl 1):33-54. French.

24. Al-Hadithy N, Papanna MC, Farooq S, Kalairajah Y. How to read a postoperative knee replacement radiograph. Skeletal Radiol. 2012;41(5):493-501. doi:10.1007/s00256-011-1297-x

25. Mullaji AB, Shah S, Shetty GM. Mobile-bearing medial unicompartmental knee arthroplasty restores limb alignment comparable to that of the unaffected contralateral limb. Acta orthopaedica. 2017;88 (1):70-74. doi:10.1080/17453674.2016.1253327

26. Kennedy WR, White RP. Unicompartmental arthroplasty of the knee. Postoperative alignment and its influence on overall results. Clin Orthop Relat Res. 1987;(221):278-285.

27. Subramanian SS, MoongilpattiSengodan M. Role of full length weight bearing radiograph in assessing the alignment in total knee arthroplasty. IOSR J Dent Med Sci. 2016;15(07):55-63. doi:10.9790/ 0853-150785563

28. Allen MM, Pagnano MW. Neutral mechanical alignment: is it necessary? Bone Joint J. 2016;98-B(1 Suppl A):81-83. doi:10.1302/ 0301-620X.98B1.36403

29. Mullaji AB, Shetty GM, Kanna R, Vadapalli RC. The influence of preoperative deformity on valgus correction angle: an analysis of 503 total knee arthroplasties. $J$ Arthroplasty. 2013;28(1):20-27. doi:10.1016/j.arth.2012.04.014

30. Park A, Stambough JB, Nunley RM, Barrack RL, Nam D. The inadequacy of short knee radiographs in evaluating coronal alignment after total knee arthroplasty. J Arthroplasty. 2016;31(4):878-882. doi:10.1016/j.arth.2015.08.015

31. Berend ME, Ritter MA, Meding JB, et al. Tibial component failure mechanisms in total knee arthroplasty. Clin Orthop Relat Res. 2004;428(428):26-34. doi:10.1097/01.blo.0000148578.22729.0e

32. Luyckx T. The Tibio-Femoral Joint Line: What is the Biomechanical and Clinical Effect of Surgical Modifications? Faculty of Medicine and Health Sciences; KU Leuven, Faculty of Medicine, Ghent Ghent University; 2015.

33. Servien E, Viskontas D, Giuffre BM, Coolican MR, Parker DA. Reliability of bony landmarks for restoration of the joint line in revision knee arthroplasty. Knee Surg Sports Traumatol Arthrosc. 2008;16(3):263-269. doi:10.1007/s00167-007-0449-y

34. Harvey WF, Yang M, Cooke TD, et al. Association of leg-length inequality with knee osteoarthritis: a cohort study. Ann Intern Med. 2010;152 (5):287-295. doi:10.7326/0003-4819-152-5-201003020-00006

35. Simsek ME, Akkaya M, Gursoy S, et al. Posterolateral overhang affects patient quality of life after total knee arthroplasty. Arch Orthop Trauma Surg. 2018;138(3):409-418. doi:10.1007/s00402017-2850-4

36. Mahoney OM, Kinsey T. Overhang of the femoral component in total knee arthroplasty: risk factors and clinical consequences. J Bone Joint Surg Am. 2010;92(5):1115-1121. doi:10.2106/JBJS.H.00434

37. Chung BJ, Kang YG, Chang CB, Kim SJ, Kim TK. Differences between sagittal femoral mechanical and distal reference axes should be considered in navigated TKA. Clin Orthop Relat Res. 2009;467 (9):2403-2413. doi:10.1007/s11999-009-0762-5

38. Dorr LD, Boiardo RA. Technical considerations in total knee arthroplasty. Clin Orthop Relat Res. 1986;(205):5-11.

39. Rassir R, van de Bunt F, Sierevelt IN, Nolte PA. The value of postoperative prosthesis alignment and patellar height measurements on standard X-rays after total knee arthroplasty: does it relate to knee function after 5years? Knee. 2019;26(1):213-221. doi:10.1016/j. knee.2018.09.014

40. Bellemans J, Banks S, Victor J, Vandenneucker H, Moemans A. Fluoroscopic analysis of the kinematics of deep flexion in total knee arthroplasty. Influence of posterior condylar offset. J Bone Joint Surg Br. 2002;84(1):50-53. doi:10.1302/0301-620X.84B1.0840050
41. Clarke HD, Hentz JG. Restoration of femoral anatomy in TKA with unisex and gender-specific components. Clin Orthop Relat Res. 2008;466(11):2711-2716. doi:10.1007/s11999-008-0454-6

42. Miller RK, Goodfellow JW, Murray DW, O'Connor JJ. In vitro measurement of patellofemoral force after three types of knee replacement. $J$ Bone Joint Surg Br. 1998;80-B(5):900-906. doi:10.1302/0301-620X.80B5.0800900

43. Eshnazarov KE, Seon JK, Song EK. Comparison of radiological assessments patellar resurfacing with retention for grade IV osteoarthritis in patellofemoral joint accomplished total knee arthroplasty. Vestn Rentgenol Radiol. 2016;97(1):28-32. doi:10.20862/0042-46762016-97-1-28-32

44. Insall J, Salvati E. Patella position in the normal knee joint. Radiology. 1971;101(1):101-104. doi:10.1148/101.1.101

45. Caton J, Deschamps G, Chambat P, Lerat JL, Dejour H. Patella infera. Apropos of 128 cases [Patella infera. About 128 cases]. Revue de chirurgie orthopedique et reparatrice de l'appareil moteur. 1982;68(5):317-325. French.

46. Sarmah SS, Patel S, Hossain FS, Haddad FS. The radiological assessment of total and unicompartmental knee replacements. J Bone Joint Surg Br. 2012;94-B(10):1321-1329. doi:10.1302/0301-620X.94B10.29411

47. Selvarajah E, Hooper G. Restoration of the joint line in total knee arthroplasty. J Arthroplasty. 2009;24(7):1099-1102. doi:10.1016/j. arth.2008.06.030

48. Gujarathi N, Putti AB, Abboud RJ, MacLean JG, Espley AJ, Kellett CF. Risk of periprosthetic fracture after anterior femoral notching. Acta Orthopaedica. 2009;80(5):553-556. doi:10.3109/ 17453670903350099

49. Lo CS, Wang SJ, Wu SS. Knee stiffness on extension caused by an oversized femoral component after total knee arthroplasty: a report of two cases and a review of the literature. J Arthroplasty. 2003;18 (6):804-808. doi:10.1016/S0883-5403(03)00331-0

50. Miller TT. Imaging of knee arthroplasty. Eur J Radiol. 2005;54 (2):164-177. doi:10.1016/j.ejrad.2005.01.020

51. Mulligan ME, Jones ED. Femoral sulcus angle measurements. Am J Orthoped. 1997;26(8):541-543.

52. Merchant AC, Mercer RL, Jacobsen RH, Cool CR. Roentgenographic analysis of patellofemoral congruence. J Bone Joint Surg Am. 1974;56(7):1391-1396. doi:10.2106/00004623197456070-00007

53. Dutton RA, Khadavi MJ, Fredericson M. Patellofemoral Pain. Phys Med Rehabil Clin N Am. 2016;27(1):31-52. doi:10.1016/j. pmr.2015.08.002

54. Gomes LS, Bechtold JE, Gustilo RB. Patellar prosthesis positioning in total knee arthroplasty. A roentgenographic study. Clin Orthop Relat Res. 1988;(236):72-81.

55. Davies AP, Costa ML, Shepstone L, Glasgow MM, Donell S. The sulcus angle and malalignment of the extensor mechanism of the knee. J Bone Joint Surg Br. 2000;82-B(8):1162-1166. doi:10.1302/ 0301-620X.82B8.0821162

56. Hsu HC, Luo ZP, Rand JA, An KN. Influence of patellar thickness on patellar tracking and patellofemoral contact characteristics after total knee arthroplasty. J Arthroplasty. 1996;11(1):69-80. doi:10.1016/ S0883-5403(96)80163-X

57. Bengs BC, Scott RD. The effect of patellar thickness on intraoperative knee flexion and patellar tracking in total knee arthroplasty. J Arthroplasty. 2006;21(5):650-655. doi:10.1016/j. arth.2005.07.020

58. Lewonowski K, Dorr LD, McPherson EJ, Huber G, Wan Z. Medialization of the patella in total knee arthroplasty. J Arthroplasty. 1997;12(2):161-167. doi:10.1016/S0883-5403(97)90062-0

59. Chia SL, Merican AM, Devadasan B, Strachan RK, Amis AA. Radiographic features predictive of patellar maltracking during total knee arthroplasty. Knee Surg Sports Traumatol Arthrosc. 2009;17 (10):1217-1224. doi:10.1007/s00167-009-0832-y 
60. Savin L, Botez P, Mihailescu D, Predescu V, Grierosu C. Preoperative radiological measurement of femoral rotation for prosthetic positioning in total knee arthroplasty. Int Orthop. 2016;40 (9):1855-1860. doi:10.1007/s00264-015-3110-2
61. Viel T, Casin C, Ducellier F, Steiger V, Bigorre N, Bizot P. Is radiographic measurement of distal femoral torsion reliable? OTSR. 2013;99(5):517-522. doi:10.1016/j.otsr.2013.02.009

\section{Publish your work in this journal}

Orthopedic Research and Reviews is an international, peer-reviewed, open access journal that focusing on the patho-physiology of the musculoskeletal system, trauma, surgery and other corrective interventions to restore mobility and function. Advances in new technologies, materials, techniques and pharmacological agents are particularly

Submit your manuscript here: https://www.dovepress.com/orthopedic-research-and-reviews-journal welcome. The manuscript management system is completely online and includes a very quick and fair peer-review system, which is all easy to use. Visit http://www.dovepress.com/testimonials.php to read real quotes from published authors. 